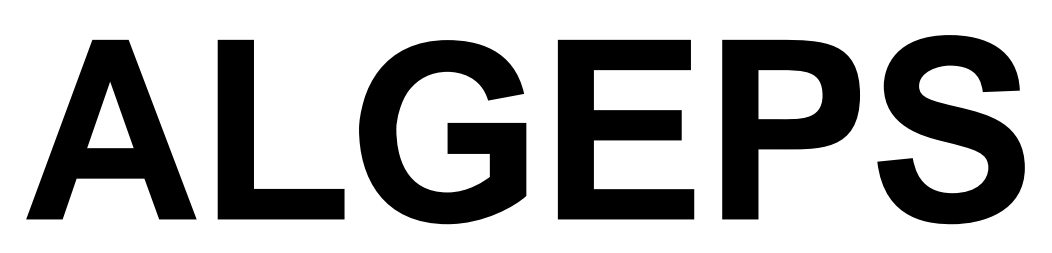

REVISTA DE GEOLOGIA, SĖRIE B no 586 - Abril del 2012

ISSN $1132-7014$

D.L.B. 28.178 - 92

14 pàgines

RECORRIDO DESDE PURROY DE LA SOLANA (RIBAGORZA) A GABASA, PERALTA DELA SAL Y CALASANZ, A TRAVÉS DEL PATRIMONIO GEOLÓGICO Y MINERO DE LAS COMARCA DE LA LITERA

Josep M. Mata-Perelló

Aquest recorregut va ésser experimentat amb docents el dia 8 DE JUNY DEL 2010 


\section{RECORRIDO DESDE PURROY DE LA SOLANA (RIBAGORZA) A GABASA, PERALTA DELA SAL Y CALASANZ, A TRAVÉS DEL PATRIMONIO GEOLÓGICO Y MINERO DE LAS COMARCA DE LA LITERA}

\section{ADVERTENCIAS PREVIAS}

Como en otros recorridos de RECONOCIMIENTO GEOLÓGICO (o de RECONOCIMIENTO GEOLÓGICO Y MINERO), el recorrido se compondrá de diversas PARADAS. En este caso serán seis.

Por otra parte, habrá que tener en cuenta, en todo momento, especialmente antes de empezar los recorridos de los diferentes tramos, el estado de los caminos y carreteras, por donde transitará el recorrido. Al respecto, cabe decir que prácticamente todos estos tramos se halla en buenas condiciones.

Finalmente, como ya hacemos en otros recorridos similares, queremos decir que hace falta tener un cuidado muy especial en el respeto a la naturaleza, a lo largo de todo el recorrido del itinerario, y también fuera de él.

\section{BREVE INTRODUCCIÓN GEOLÓGICA}

Como en el caso de los itinerarios geológicos: DE BINEFAR A ALINS o de ALTORRICÓN A NACHÁ o el de ALBELDA A CAMPORRELS, el recorrido de este itinerario, se desarrollará exclusivamente por dos de las unidades geológicas que constituyen el subsuelo aragonés: la Depresión Geológica del Ebro (por la que circularemos en los tramos iniciales, desde Purroy de la Solana hasta las inmediaciones de Peralta de la Sal) y los Pirineos, (por donde circularemos en los tramos finales del recorrido, entre la última población antes mencionada y Calasanz).

Así, a lo largo de todo de todo el primer tramo del recorrido del itinerario (dentro de la Depresión Geológica del Ebro) se irán encontrando inicialmente afloramientos cenozoicos, en su mayoría de carácter detrítico, pertenecientes al Oligoceno.

Luego, ya dentro del recorrido por los Pirineos, se irán encontrando afloramientos triásicos del Keuper (con niveles arcillosos y yesosos). También predominaran los afloramientos de los materiales carbonatados del Cretácico, dentro de los afloramientos pirenaicos de las Sierras Marginales, en el denominado Pirineo Meridional. Asimismo, a menudo, encontraremos afloramientos de los materiales detríticos cuaternarios, por encima de los anteriores 


\section{BREVE INTRODUCCIÓN GEOGRÁFICA}

En este caso, el recorrido del presente itinerario se efectuará casi exclusivamente por una comarca aragonesa, concretamente la de la Litera / Llitera. Sin embargo, el recorrido se iniciará en Purroy de la Solana, dentro de la comarca de la Ribagorza. Luego, el recorrido penetrará en la Litera / Llitera, cerca de Gabasa, no abandonándose ya esta comarca hasta llegar a Calasanz, en donde terminará.

En este recorrido, se circulará por los términos municipales de: Benabarre (de la Ribagorza, a cuyo municipio pertenece Purroy de la Solana), Peralta - Calasanz Gabasa, perteneciente a la comarca de la Litera / Llitera, en donde finalizará el recorrido.

Por lo que concierne a la Litera / Llitera, como es conocido esta comarca, gravita en torno a dos ríos de la misma cuenca: el Noguera Ribagorzana que drena los sectores orientales y el Cinca que hace lo propio con los sectores occidentales. Por lo que concierne al recorrido de este itinerario, discurrirá por los sectores centrales de interfluvio de las dos cuencas.

\section{OBJETIVOS GENERALES DE ESTE ITINERARIO}

En este itinerario, los objetivos generales que se han de conseguir, se pueden concretar en los siguientes aspectos:

1.- Estudio y reconocimiento de los materiales cenozoicos (en buena parte del Oligoceno y del Mioceno, según los lugares) situados en la Depresión Geológica del Ebro, que iremos encontrando a lo largo del recorrido del itinerario, entre la población de Purroy de la Solana y la inmediaciones de Peralta de la Sal. En buena parte estos materiales son de carácter detrítico.

2.- Estudio y reconocimiento de los materiales mesozoicos (en buena parte del Triásico y del Cretácico) situados en las Sierras Marginales Pirenaicas, que iremos encontrando a lo largo del recorrido del itinerario, entre Peralta de la Sal y Calasanz, fundamentalmente.

3.- Visión de algunas de las antiguas explotaciones encontradas a lo largo del recorrido del itinerario. En concreto de:

3A) de las explotaciones de minerales de manganeso, situadas en las cercanías de Gabasa entre los afloramientos cenozoicos.

3B) de las explotaciones de sal común que encontraremos en las cercanías de Peralta de la Sal y de Calasanz.

4.- Observación del impacto producido por las actividades mineras. I si se da el caso, de las restauraciones realizadas para paliar este impacto.

5.- Visión de los diferentes lugares directamente relacionados con el Patrimonio Geológico y Minero que iremos encontrando a lo largo del recorrido del presente itinerario. Dentro del primero, veremos el congosto situado en el Barranco del Molino, 
cerca de Gabasa. Y dentro del segundo nos referimos a las importantes salinas de Peralta de la Sal y de Calasanz.

\section{ANTECEDENTES BIBLIOGRÁFICOS}

En relación con este itinerario, no conocemos ningún antecedente, relativo a otro itinerario que discurra por este lugar. En este sentido, este itinerario ya constituye un antecedente, si no estamos equivocados. Existen antecedentes en itinerarios cercanos nuestros: MATA - PERELLÓ (1990, 1991, 1996 t 2002); así como en MATA PERELLÓ y MONTANÉ GARCÍA (2002 y 2004). Aunque se trata de recorridos no coincidentes con el que ahora presentamos.

Por otra parte, haremos mención de algunos trabajos, de carácter geológico generalista, que corresponden a los trabajos del IGME (1972, 1974 y 1975), relativos al Mapa Geológico de España (a Escala 1.200.000), al Mapa Metalogenético de España y al Mapa de Rocas Industriales de España También cabe mencionar a GUIMERÁ et altri (1992), así como a RIBA et altri (1976).

Con respecto a las mineralizaciones que iremos encontrando, mencionaremos los trabajos de: CALVO et altri (1988); MAESTRE (1845); así como nuestros trabajos: MATA-PERELLÓ (1987 y 1998).

También mencionaremos el trabajo de PRAMES (2005) dedicado a la comarca del Campo de Belchite. Así como el del GOBIERNO DE ARAGÓN (2001), dedicado a los Puntos de Interés Geológico de Aragón.

Finalmente, diremos que todos estos trabajos (así como otros que ahora no hemos aludido), figurarán mencionados, por orden alfabético, en el apartado dedicado a las REFERENCIAS BIBLIOGRÁFICAS.

\section{RECORRIDO DEL ITINERARIO}

Este recorrido se iniciará en las inmediaciones de la población de Purroy de la Solana (perteneciente al municipio de Benabarre, en la comarca de la Ribagorza) en donde se efectuará la primera parada. Tras ello, el recorrido se dirigirá hacía la cercana población de Gabasa, en donde se realizaran dos paradas.

Posteriormente, el recorrido se dirigirá hacía Peralta de la Sal, cerca de la cual se efectuaran dos paradas más. Tras ello, el recorrido se dirigirá hacía Calasanz, en donde finalizará después de realizarse dos paradas más.

\section{DESCRIPCIÓN DEL ITINERARIO}

Como de costumbre, haremos una serio de PARADAS (o ESTACIONES), en donde se realizaran diversas explicaciones en torno a las características del lugar en donde se halla la PARADA. Por otra parte, en ellas haremos mención del término municipal dónde se encuentran, así como del número del "Mapa Topográfico Nacional 
(a escala 1:50.000, que indicaremos entre paréntesis. Así, ahora (en este recorrido) utilizaremos solamente tres hojas, concretamente las siguientes: 288 (o de Fonz), 326 (o de Monzón) y 327 (o de Os de Balaguer). Así, la relación ordenada de las paradas que constituyen el recorrido de este itinerario, es la siguiente:

PARADA 1. POLJÉ DE PURROY DE LA SOLANA, (Purroy de la Solana, término municipal de Benabarre, comarca de la Ribagorza). (Hoja 288).

El recorrido del itinerario cabe iniciarlo en este lugar, en las inmediaciones de Purroy de la Solana, sobre la carretera nacional N-230. Al llegar a las inmediaciones del cruce de la carretera que se dirige (por la derecha hacía el pueblecito de lo Lloc, podrá hacerse la primera parada.

En este recorrido, desde Purroy hasta el cruce, hemos ido encontrando los materiales citados en la parada anterior, sin embargo, al encarar la recta que conduce a Purroy de la Solana, veremos una gran zona deprimida. Analizándola bien veremos que se trata de una depresión de origen kárstico, el que hemos denominado como Poljé de Purroy de la Solana.

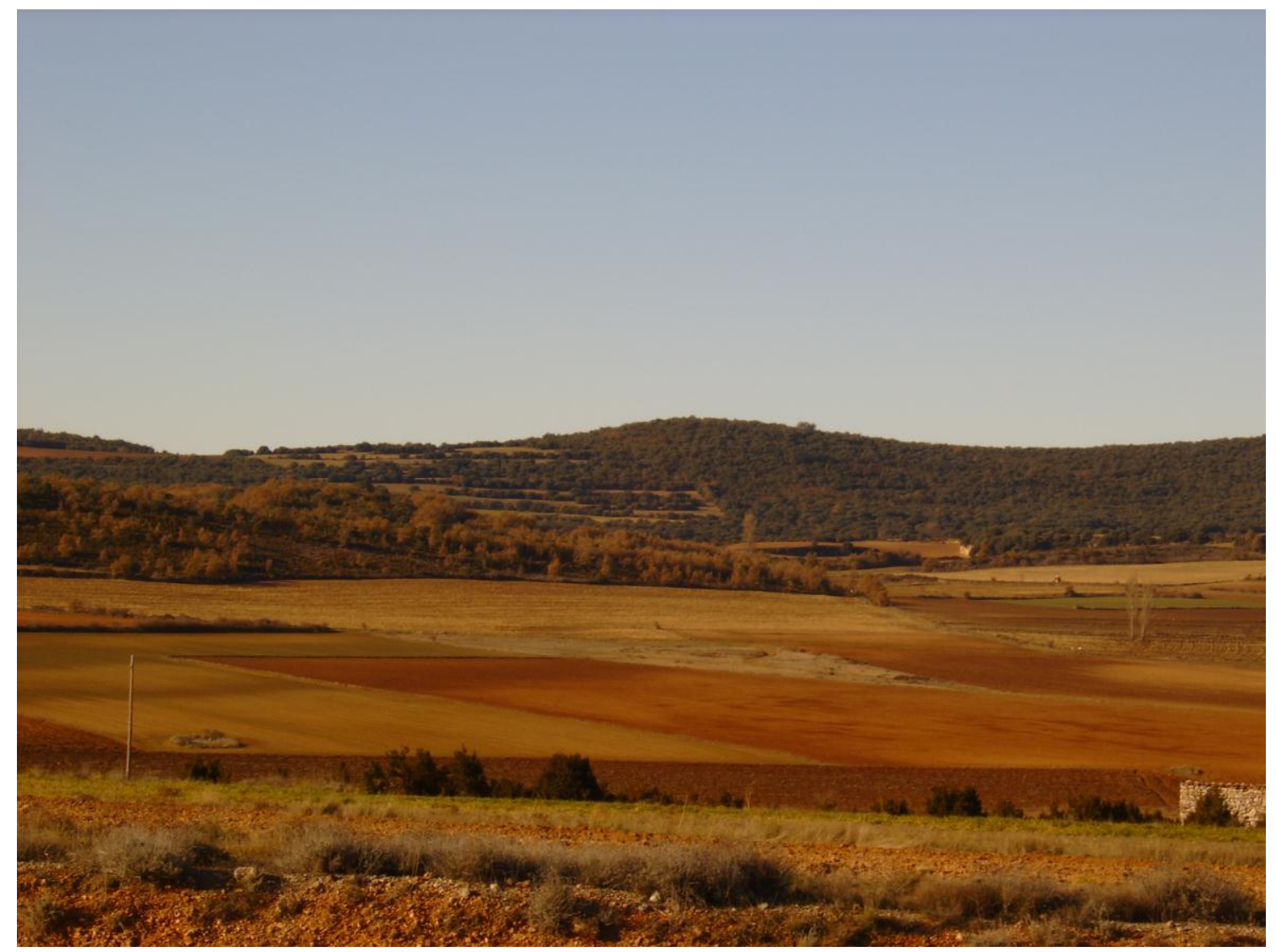

Aspecto del extenso Poljé de Purroy de la Solana 
Cabe señalar, que este poljé puede verse muy bien desde el núcleo antiguo de Purroy de la Solana. En cualquier caso, es visible desde diversos lugares la extensa depresión, así como los típicos materiales rojizos que aparecen por doquier.

PARADA 2. MINA DE MANGANESO DE GABASA, MINA DE SANTA TERESA, (Gabasa, término municipal de Peralta - Calazanz - Gabasa, comarca de la Litera / Llitera). (Hoja 288).

Tras realizar la parada anterior, es necesario efectuar un recorrido de unos $6 \mathrm{Km}$ con la finalidad de llegar hasta las inmediaciones de Gabasa, concretamente al barranco de la Talcerva. Al llegar a él tomaremos un sendero que nos conducirá hasta las Minas de Manganeso de Gabasa. Para ello, desde la parada anterior habremos recorrido unos 6 $\mathrm{Km}$.

En este recorrido habremos ido encontrando afloramientos de los materiales detríticos cenozoicos, de carácter postororogénico. Estos son también los materiales que aparecen en la antigua mina.

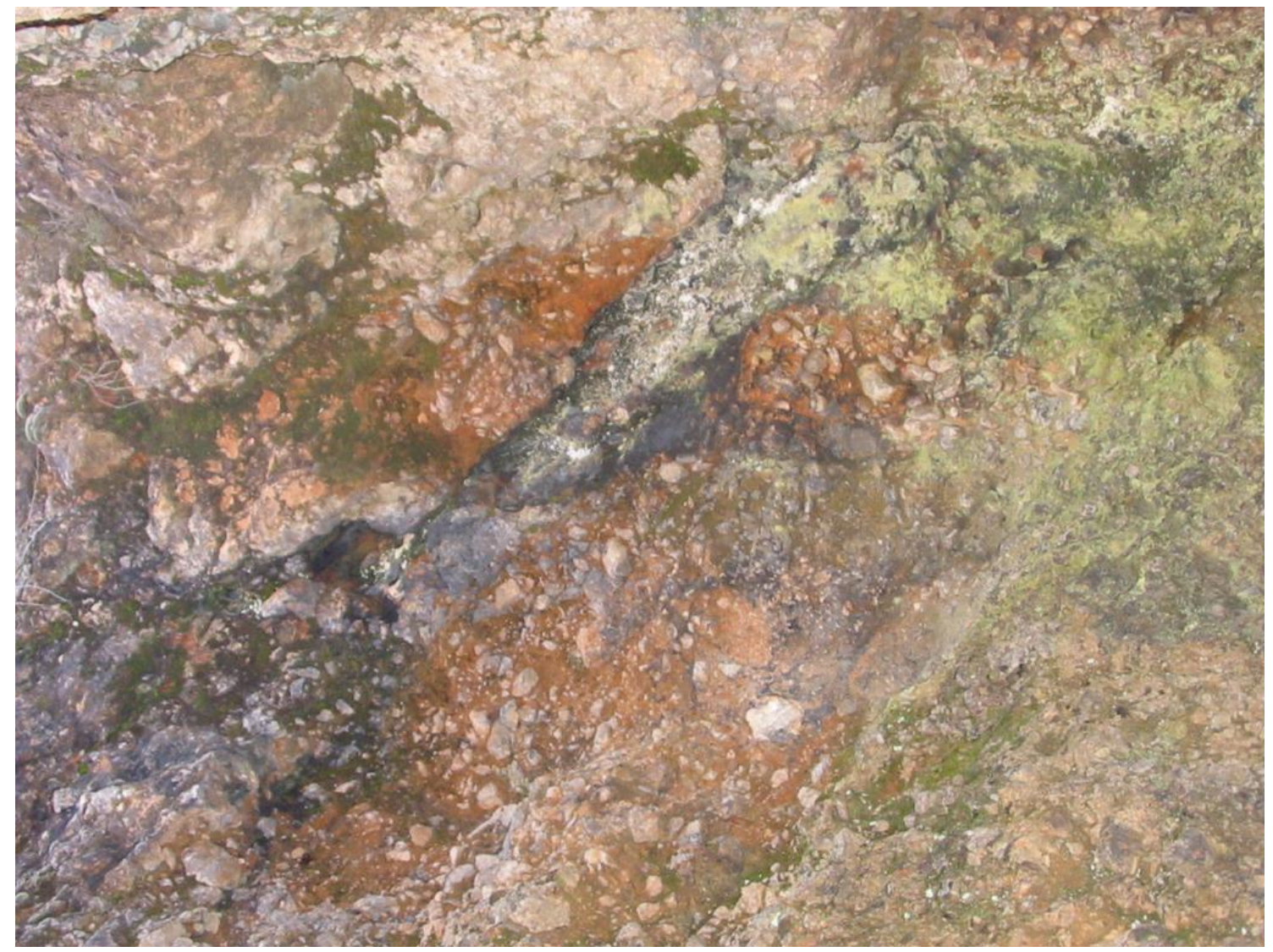

Detalle de la mineralización

En este lugar, entre los materiales cenozoicos, hay una interesante mineralización. Así, los minerales de manganeso se hallan diseminados entre los materiales detríticos. Entre otros minerales de manganeso se encuentran: PIROLUSITA, PSILOMELANA, RAMSDELLITA, TODOROGUITA y MANGANOCALCITA, 
Asimismo, se encuentran también minerales de hierro con GOETHITA (limonita), HEMATITES. Entre otros minerales, cabe citar a la CALCITA

PARADA 3. BARRANCO DE GABASA, (Gabasa, término municipal de Peralta - Calazanz - Gabasa, comarca de la Litera / Llitera). (Hoja 288).

Después de realizar la parada anterior, conviene retornar a la carretera que conduce a Gabasa y a Peralta de la Sal. Al llegar al primer pueblo, será conveniente atravesarlo a pie, hasta llegar al barranco. Ahí realizaremos una nueva parada, tras haber recorrido unos $2 \mathrm{Km}$ escasos, desde la mina de manganeso.

En este recorrido habremos encontrado afloramientos de los materiales detríticos oligocénicos mencionados en la parada anterior. Sin embargo, cerca del pueblo de Gabasa habremos atravesado un imponente farallón de calizas mesozoicas del Cretácico. Se trata de una capa verticalizada que forma parte de un pliegue tumbado. Se trata de un gran anticlinal surcado en su centro por el barranco de Gabasa.

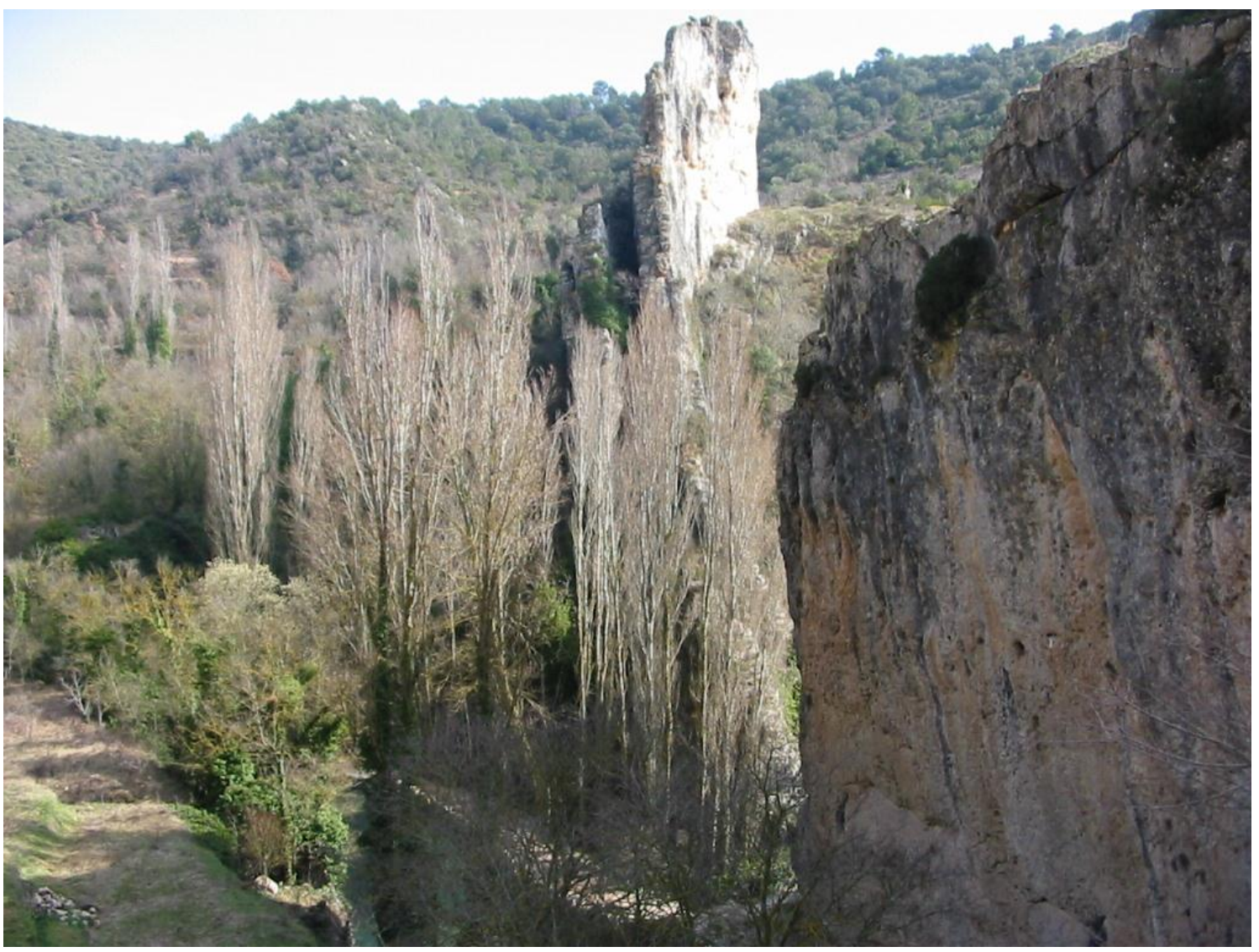

Un aspecto del farallón de calizas de un flanco del anticlinal.

Este importante elemento estructural forma parte del Patrimonio Geológico de la comarca de la Litera.

Como un hecho importante a considerar, está el de que la población se halla al SW de este farallón, con lo cual se halla perfectamente resguardada de los vientos procedentes del norte. 
PARADA 4. SALINAS DE PERALTA DE LA SAL, (Peralta de la Sal, término municipal de Peralta - Calazanz - Gabasa, comarca de la Litera / Llitera). (Hoja 327).

Después de efectuar la parada anterior en Gabasa, conviene retornar a la carretera que ahora conduce a Peralta de la Sal. Poco antes de llegar a la población (a menos de unos 100 metros) encontraremos el rótulo que indica el camino para ir a las salinas, a la izquierda de la carretera. En poco más de $1 \mathrm{Km}$ llegaremos al inicio de las mismas, para poderlas recorrer a pie. Ahí efectuaremos una nueva parada, a unos $5 \mathrm{Km}$ de la realizada en Gabasa.

En este recorrido habremos ido encontrando afloramientos de los materiales mesozoicos citados en la parada anterior. Estos materiales pertenecen a las Sierras Marginales Pirenaicas, en donde estamos ahora casi plenamente situados. Por otra parte, cerca de Peralta habremos ido encontrando afloramientos de los materiales triásicos del Keuper, con abundantes niveles de arcillas y de yesos. Entre estos últimos se encuentran pasadas de HALITA.

Sin embargo, al llegar al inicio de las salinas, habremos encontrado unos niveles verticalizados del Eoceno. Es decir, hemos vuelto a entrar en la Depresión Geológica del Ebro. Así habremos visto niveles de yesos, arcillas y lutitas. Las eras de las salinas se encuentran sobre estos materiales, aunque el agua salada procede del cercano Keuper, de donde se extraía el agua salada por pozos.

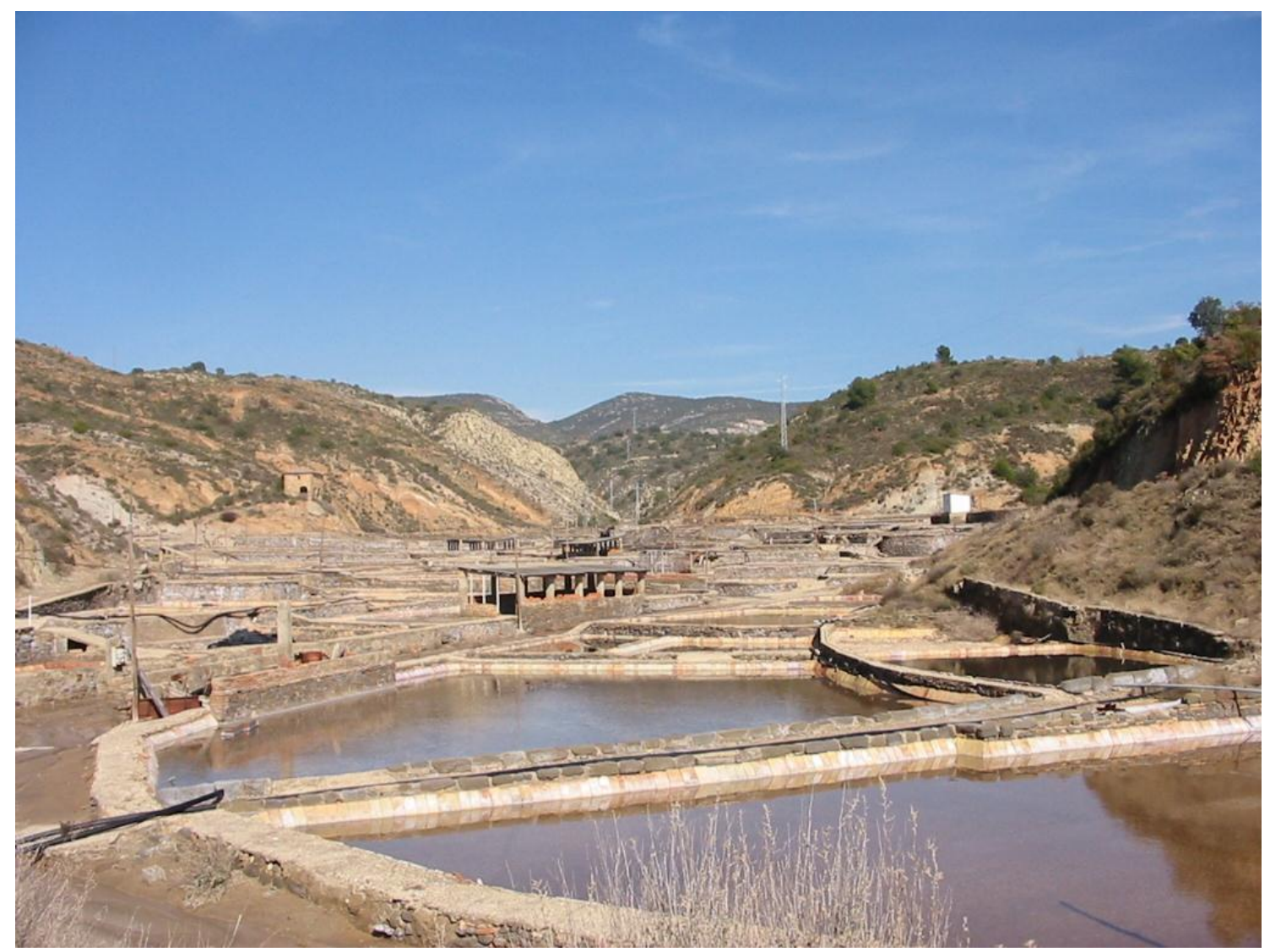

Las salinas, sobre los materiales del Eoceno. Al fondo del barranco se ve el Keuper 
La sal obtenida (HALITA) se halla interestratificada entre los materiales triásicos del Keuper. Así, al circular las aguas superficiales por estos materiales se salinizan al disolver la sal. Luego, al recogerse esta agua en las eras se evapora el agua, obteniéndose la HALITA. Previamente, en las balsas ya se han decantado los sulfatos que también llevan esta agua.

Estas importantes salinas, actualmente inactivas, constituyen un elemento muy importante del Patrimonio Minero de la comarca de la Litera.

PARADA 5. PLAYA FÓSIL, (Peralta de la Sal, término municipal de Peralta - Calazanz - Gabasa, comarca de la Litera / Llitera). (Hoja 327).

Después de realizar la parada anterior, conviene retornar a la carretera, para cruzar el pueblo de Peralta de la Sal. Luego será necesario continuar por la carretera que conduce a Azanuy y a Calasanz. Poco después de tomar esta carretera será necesario seguir por un camino que nos conducirá a la Playa Fósil. Éste lugar se halla a unos 100 metros de la carretera. Ahí efectuaremos una nueva parada, a unos $4 \mathrm{Km}$ más allá de la anterior.

En este recorrido, hemos vuelto a atravesar los materiales cenozoicos (fundamentalmente del Oligoceno) de la Depresión Geológica del Ebro, en donde estamos de nuevo. Así, se van viendo afloramientos de niveles calcolutíticos y arenosos (como en el lugar de la parada).

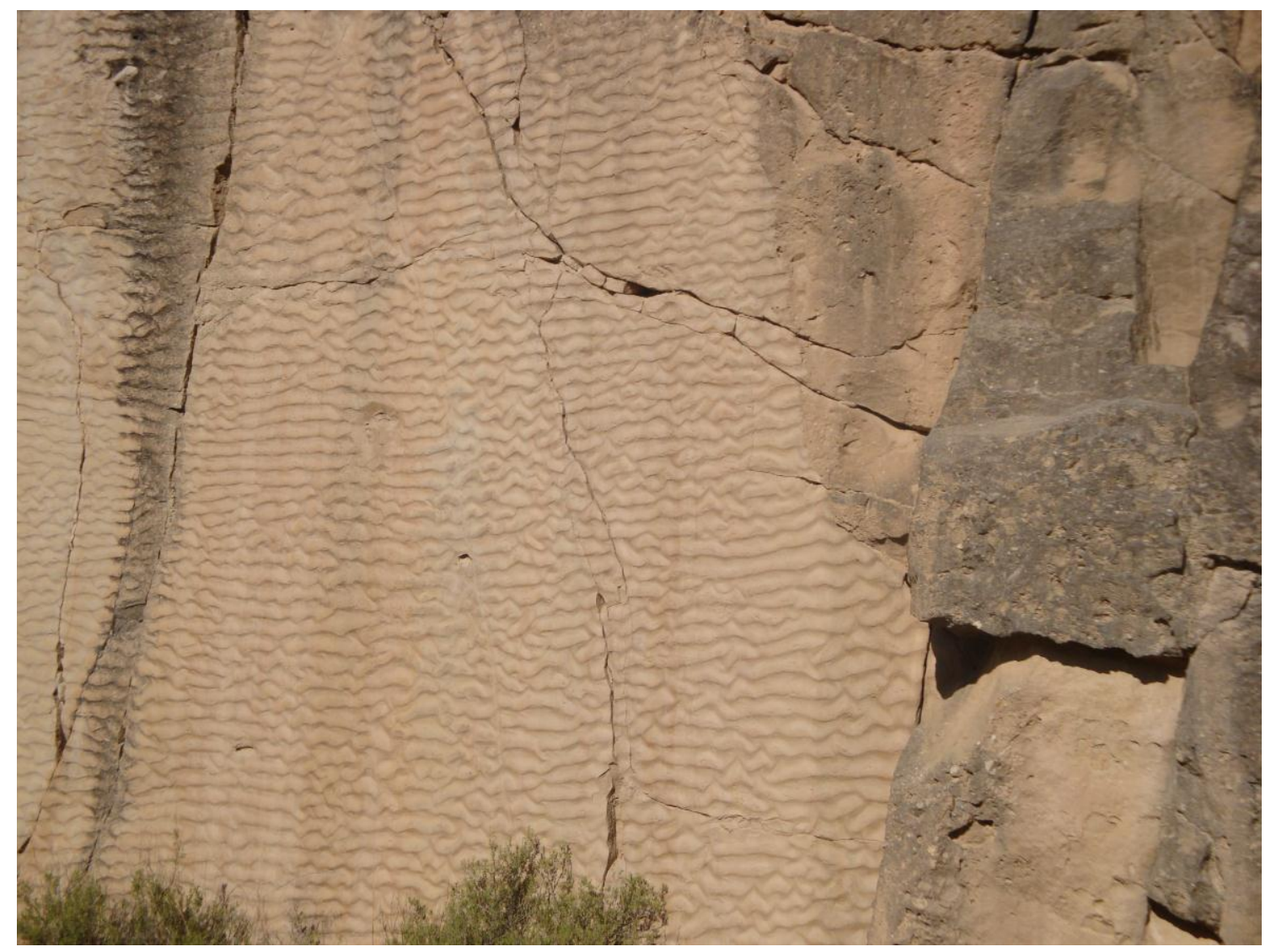

Un aspecto de la playa fósil, sobre afloramientos arenosos del Oligoceno 
Evidentemente, se trata de un buen ejemplo de ondulitas o "ripple - marks". Constituye un elemento importante del Patrimonio Geológico de la comarca de la Litera.

PARADA 6. SALINAS DE CALAZANZ, (Calasanz, término municipal de Peralta - Calazanz - Gabasa, comarca de la Litera / Llitera). (Hoja 288).

Tras efectuar la parada anterior, conviene continuar el recorrido por la carretera que conduce a Azanuy y a Calasanz (dejando luego el ramal que va al primer pueblo). Al llegar al segundo, nos convendrá tomar el camino que conduce hacía las salinas, Ahí efectuaremos una nueva parada, tras un recorrido próximo a los $4 \mathrm{Km}$.

En este recorrido, hemos ido encontrando los materiales citados en la parada anterior. Luego, al llegar a los alrededores de las salinas, habremos vuelto a encontrar afloramientos de los materiales carbonatados mesozoicos. Pero sobretodo, entorno a las salinas habremos encontrado de nuevo afloramientos de las arcillas y yesos del Triásicos. Es decir: en este tramo final del recorrido, hemos vuelto a entrar dentro de las Sierras Marginales Pirenaicas en donde estamos ahora.

En este lugar hay unas importantes salinas, cuyas eras están sobre los afloramientos triásicos. Junto a ellas hay un pozo del que se extraía el agua salada entre los materiales del Keuper.

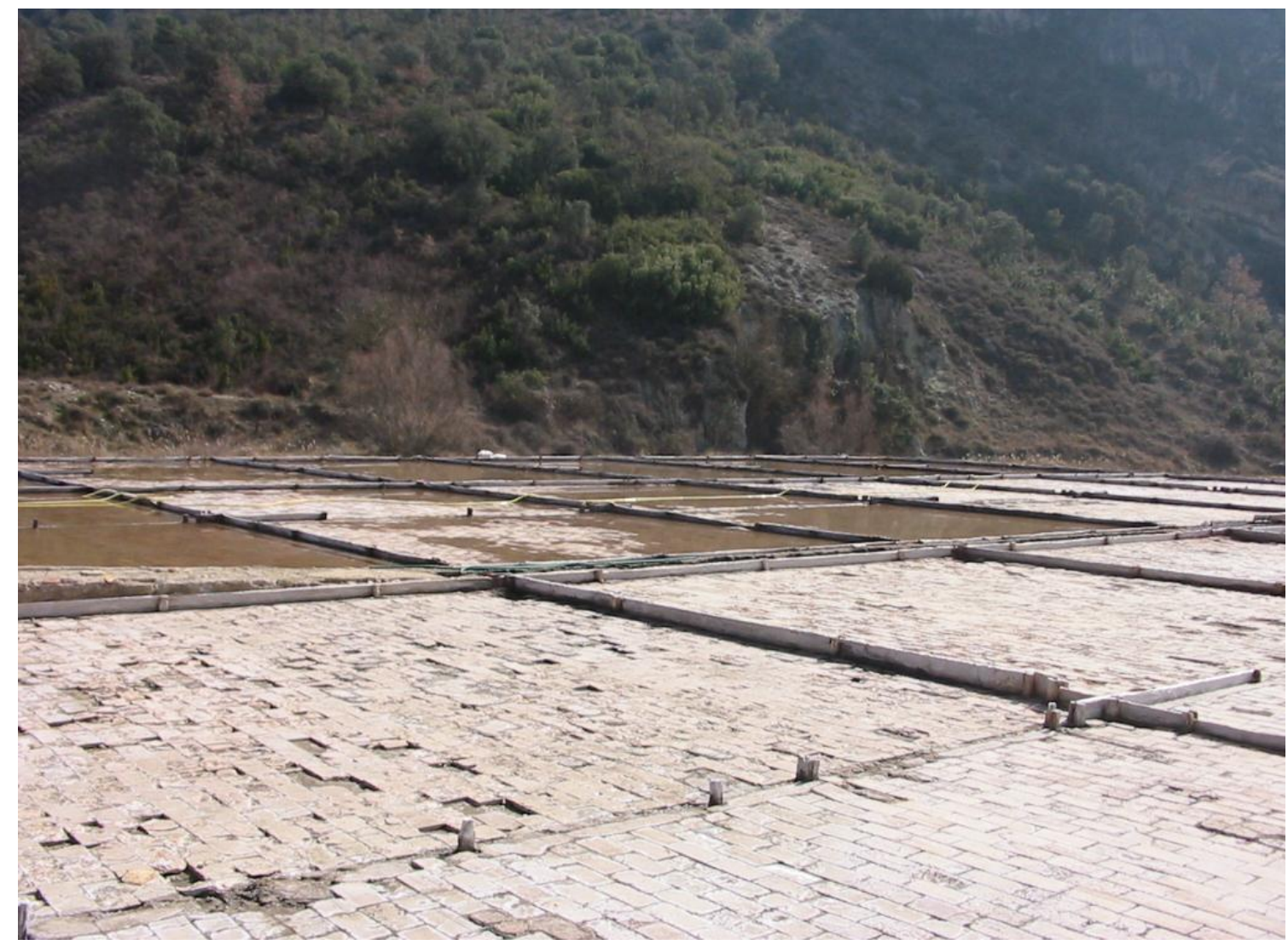

Eras de las salinas 
Estas salinas constituyen un elemento importante del Patrimonio Minero de la comarca de la Litera

Por otra parte, muy cerca de las salinas se halla el Pozo de Chelo (Pozo de Hielo), en donde podíamos efectuar una parada complementaria de este recorrido, si fuera necesario (PARADA 6 - BIS). Se trata de un elemento minero muy bien conservado.

PARADA 7. BARRANCO DE CALASANZ, (Calasanz, término municipal de Peralta - Calazanz - Gabasa, comarca de la Litera / Llitera). (Hoja 288).

Después de efectuar la parada anterior, conviene efectuar un breve recorrido a pie desde las salinas, por el barranco que conduce hacía el Sur. Así, dentro de este. Efectuaremos la última parada de este itinerario, habiendo recorrido $1 \mathrm{Km}$ a pie.

En este recorrido hemos ido transitando por entre los afloramientos de los materiales carbonatados cretácicos de las Sierras Marginales Pirenaicas, en donde estamos ahora. En este lugar, las aguas del barranco han formado un pequeño congosto.

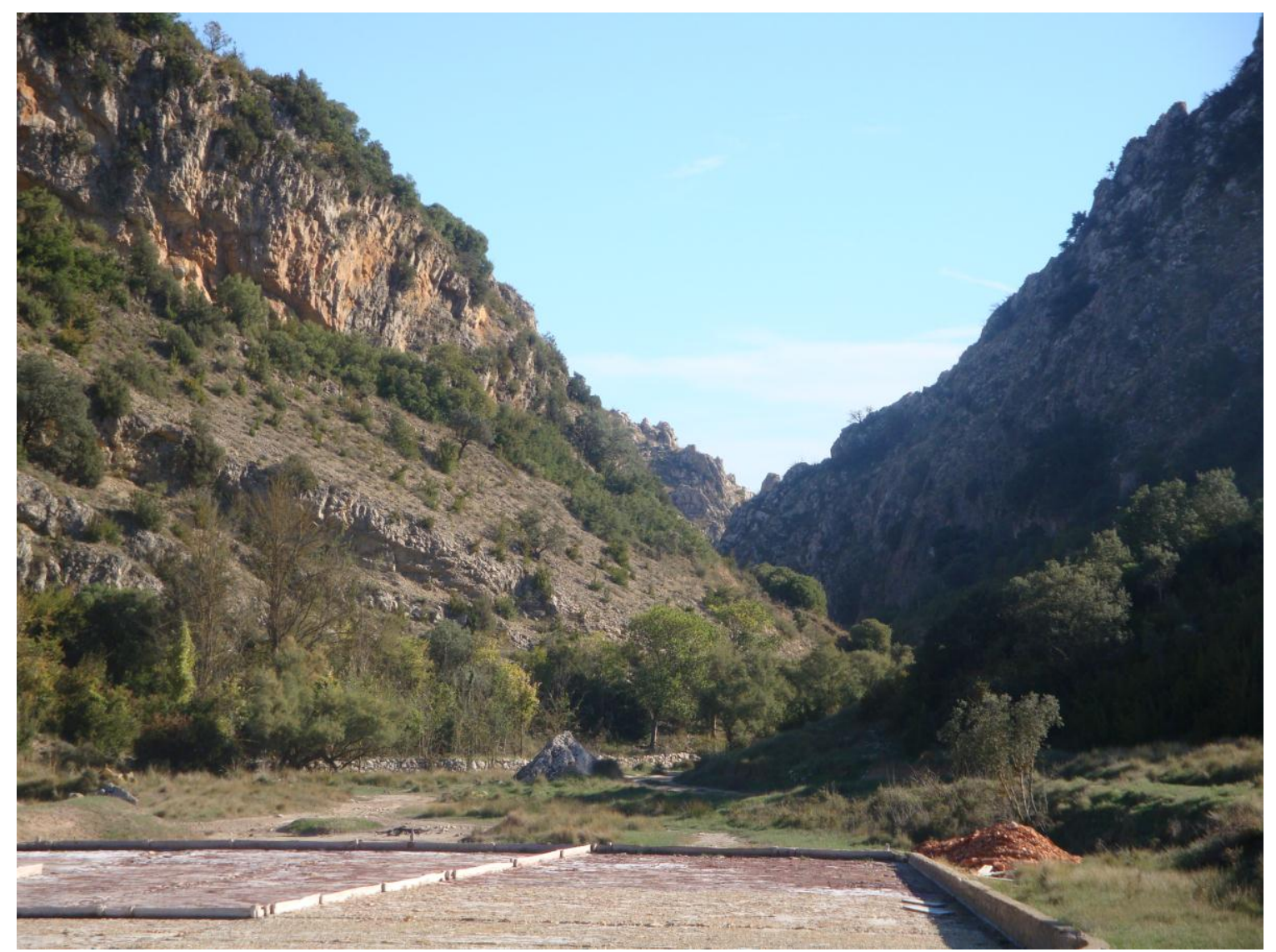

Las salinas con el congosto al fondo 


\section{EN ESTE LUGAR FINALIZA EL ITINERARIO}

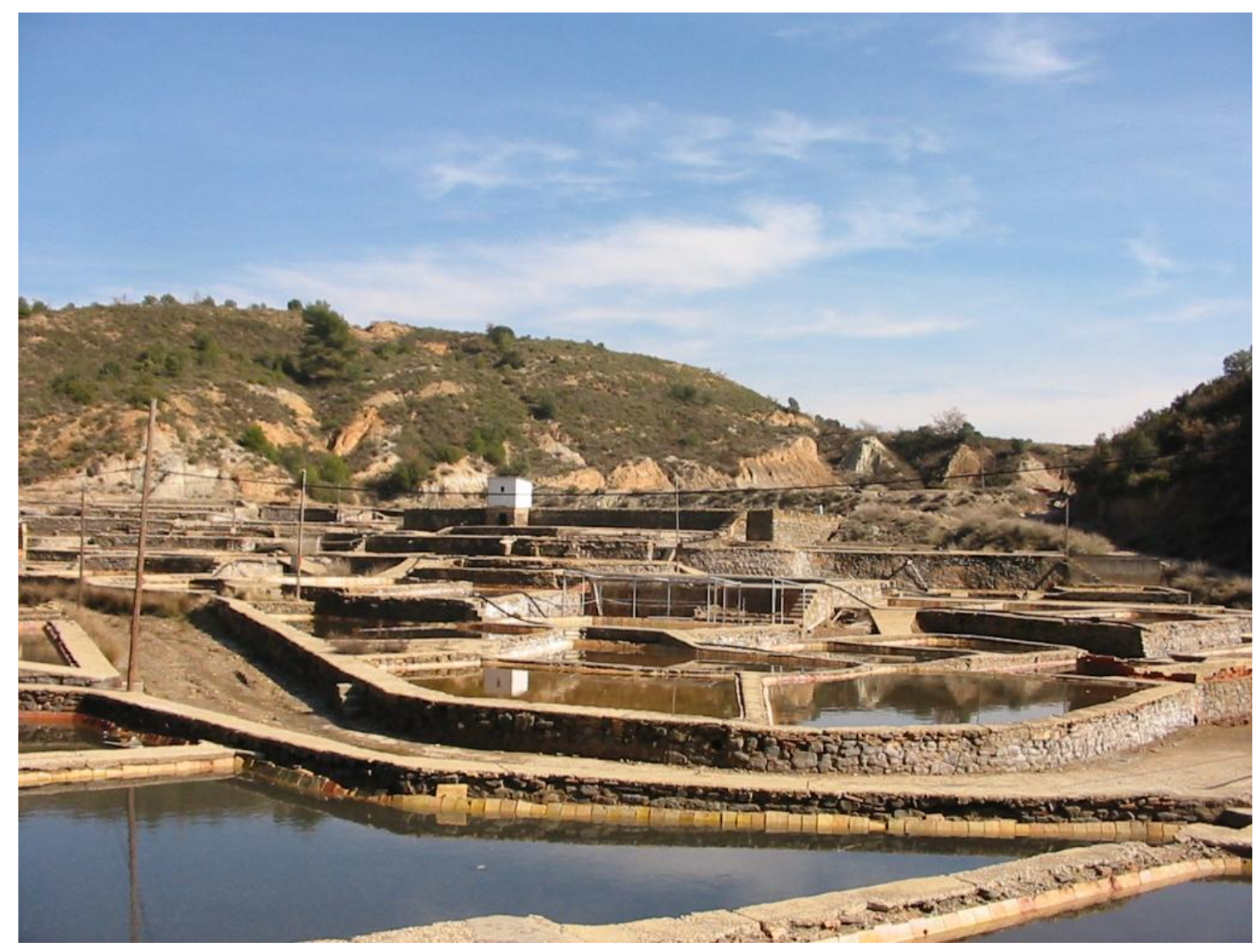

SALINAS DE PERALTA DE LA SAL

\section{BIBLIOGRAFÍA}

CALVO, M. et altri (1988).- Minerales de Aragón. Colección Temas geológicos. 200 pag. Zaragoza

GOBIERNO DE ARAGÓN (2001).- Puntos de Interés Geológico de Aragón. Consejería de Medio Ambiente del Gobierno de Aragón. Zaragoza

GUIMERÀ, J. et altri (1992).- Geología (II), Historia Natural de los Países Catalanes, Volum.2, 547 pag. Enciclopèdia Catalana, S.A. Barcelona 
IGME (1972).- Mapa Geológico de España a escala 1:200.00 (Síntesis de la Cartografía existente). Hoja y memoria no 23 (Huesca). Inst. Geol. Min. España. Minist. Industria. Madrid

IGME (1974).- Mapa Metalogenético de España a escala 1:200.00. Hoja y memoria $\mathrm{n}^{\circ}$ 23 (Huesca). Inst. Geol.Min. España. Minist. Industria. Madrid

IGME (1975).- Mapa de Rocas Industriales de España a escala 1:200.00. Hoja y memoria no 23 (Huesca). Inst. Geol.Min. España. Minist. Industria. Madrid

MAESTRE, A. (1845).- Visita al Distrito Minero de Aragón y Cataluña, Anales de Minas, tomo III, 145 pag. Madrid

MALLADA, L. (1881).- Descripción física y geológica de la provincia de Huesca.Mem. Com. Mapa Geol. De España. Vol. 15. pp. 1 - 439. Madrid

MATA - PERELLÓ, J.; (1987).- Introducción al conocimiento de las mineralizaciones aragonesas. Mineralogistes de Catalunya, t.III, pp. 258-265. Barcelona

MATA - PERELLÓ, J.M. (1990).- Inventario Mineralógico de la Región del Cinca (sectores Orientales). Rodeno, 19. 36 pag. Manresa

MATA-PERELLÓ, J.M. (1991).- Selección de Itinerarios de recerca mineralógica: por la Noguera, la Litera / Llitera i la Baja Ribagorza / Baixa Ribagorça. Xaragall, 27, 36 pag. Manresa

MATA-PERELLÓ, J.M. (1996).- Recerca geològica i mineralògica per les comarques del Segrià, Noguera, Litera / Llitera y Baja Ribagorza / Baixa Ribagorça: des d'Alfarràs a Peralta de la Sal, por Estopiñan / Estopanyà. Inédito, 10 pág. Manresa

MATA-PERELLÓ, J.M. (2002).- Recorregut de recerca geològica i mineralògica per les comarques del Segrià, de la Litera / Llitera i de la Baja Ribagorza / Baixa Ribagorça: des d'Alfarràs a Purroi y en Benabarre, revista Xaragall serie $\mathrm{B}, \mathrm{n}^{\circ}$ 236, 12 pag. Manresa

MATA-PERELLÓ, J.M. i MONTANÉ GARCÍA. P. (2002).- Recorregut de recerca geològica i mineralògica per les comarques del Segrià, la Litera / Llitera i la Baja Ribagorza / Baixa Ribagorça: des d’Alfarràs a Camporrells, a Caladrones / Caladrons i a Benabarre, Inédito, 14 pag. Manresa

MATA-PERELLÓ, J.M. i MONTANÉ GARCÍA. P. (2004).- Recorregut de recerca geològica i mineralògica per les comarques del Segrià, la Litera / Llitera i la Baja Ribagorza / Baixa Ribagorça: des d'Alfarràs a Estopiñan / Estopanyà i a Benabarre, Inédito, 14 pag. Manresa

MATA-PERELLÓ, J.M. i SANZ BALAGUÉ, J. (1989).- Inventari Mineralògic de la comarca de la Litera / Llitera. Revista Tierra Endins, n 2, 32 pag. Manresa

PRAMES (2005).- Campo de Belchite. Colección RUTASCAL por Aragón. Prames, Gobierno de Aragón. 119 pag. Zaragoza. 
RIBA, O. et altri (1976).- Geografía Física de los Países Catalanes, Edit. Ketres, 254 páginas. Barcelona 